\title{
Steiner Triple Systems Intersecting in Pairwise Disjoint Blocks
}

\author{
Yeow Meng Chee \\ Netorics Pte Ltd \\ 130 Joo Seng Road \\ \#05-02 Olivine Building \\ Singapore 368357 \\ ymchee@alumni.uwaterloo.ca
}

Submitted: Feb 18, 2003; Accepted: Mar 12, 2004; Published: Apr 2, 2004

\begin{abstract}
Two Steiner triple systems $(X, \mathcal{A})$ and $(X, \mathcal{B})$ are said to intersect in $m$ pairwise disjoint blocks if $|\mathcal{A} \cap \mathcal{B}|=m$ and all blocks in $\mathcal{A} \cap \mathcal{B}$ are pairwise disjoint. For each $v$, we completely determine the possible values of $m$ such that there exist two Steiner triple systems of order $v$ intersecting in $m$ pairwise disjoint blocks.
\end{abstract}

\section{Introduction}

A set system is a pair $(X, \mathcal{A})$, where $X$ is a finite set of points, and $\mathcal{A}$ is a set of subsets of $X$, called blocks. Let $\mathcal{K}$ be a set of positive integers. The set $\mathcal{K}$ is a set of block sizes for $(X, \mathcal{A})$ if $|A| \in \mathcal{K}$ for every $A \in \mathcal{A}$.

Let $(X, \mathcal{A})$ be a set system, and let $\mathcal{G}=\left\{G_{1}, \ldots, G_{s}\right\}$ be a partition of $X$ into subsets, called groups. The triple $(X, \mathcal{G}, \mathcal{A})$ is a group divisible design (GDD) when every 2 -subset of $X$ not contained in a group appears in exactly one block and $|A \cap G| \leq 1$ for all $A \in \mathcal{A}$ and $G \in \mathcal{G}$. We denote a $\operatorname{GDD}(X, \mathcal{G}, \mathcal{A})$ by $\mathcal{K}$-GDD if $\mathcal{K}$ is a set of block sizes for $(X, \mathcal{A})$. The group type, or simply type of a $\operatorname{GDD}(X, \mathcal{G}, \mathcal{A})$ is the multiset $[|G| \mid G \in \mathcal{G}]$. When more convenient, we use the exponential notation to describe the type of a GDD: A GDD of type $g_{1}^{t_{1}} \cdots g_{s}^{t_{s}}$ is a GDD where there are exactly $t_{i}$ groups of size $g_{i}$. If $\mathcal{G}$ is not specified in a $\operatorname{GDD}(X, \mathcal{G}, \mathcal{A})$, it is taken that all groups are of size one: $\mathcal{G}=\{\{x\} \mid x \in X\}$. A $\{3\}$-GDD of type $1^{v}$ is a Steiner triple system of order $v$, and is denoted by $\operatorname{STS}(v)$.

Two GDDs $\mathcal{D}_{1}=\left(X, \mathcal{G}_{1}, \mathcal{A}_{1}\right)$ and $\mathcal{D}_{1}=\left(X, \mathcal{G}_{2}, \mathcal{A}_{2}\right)$ of the same type are said to intersect in $m$ blocks if $\left|\mathcal{A}_{1} \cap \mathcal{A}_{2}\right|=m$. If in addition, the blocks in $\mathcal{A}_{1} \cap \mathcal{A}_{2}$ are pairwise disjoint (that is, for any $A, A^{\prime} \in A_{1} \cap A_{2}, A \neq A^{\prime}$, we have $A \cap A^{\prime}=\emptyset$ ), then $\mathcal{D}_{1}$ and $\mathcal{D}_{2}$ 
are said to intersect in $m$ pairwise disjoint blocks. Define

$$
\begin{aligned}
\operatorname{Int}(T) & =\{m \mid \exists \text { two }\{3\} \text {-GDDs of type } T \text { intersecting in } m \text { blocks }\} \text {; and } \\
\operatorname{Int}_{\mathrm{d}}(T) & =\{m \mid \exists \text { two }\{3\} \text {-GDDs of type } T \text { intersecting in } m \text { pairwise disjoint blocks }\} .
\end{aligned}
$$

The intersection problem and disjoint intersection problem for $\{3\}$-GDDs of type $T$ is to determine $\operatorname{Int}(T)$ and $\operatorname{Int}_{\mathrm{d}}(T)$, respectively. A pair of GDDs is said to be disjoint if they intersect in no blocks.

The intersection problem for Steiner triple systems was completely solved by Lindner and Rosa [6]. The intersection problem for $\{3\}$-GDDs of type $2^{t}$ has also been solved by Hoffman and Lindner [5]. The case of $\{3\}$-GDDs of type $g^{3}$, which are equivalent to Latin squares of side $g$, has been settled by $\mathrm{Fu}$ [4]. Butler and Hoffman [2] finally put the intersection problem for $\{3\}$-GDDs of type $g^{t}$ to rest with the following result.

Let $b\left(g^{t}\right)=g^{2} t(t-1) / 6$ (the number of blocks in a $\{3\}$-GDD of type $g^{t}$ ), and denote by $\mathcal{I}\left(g^{t}\right)=\left\{0,1 \ldots, b\left(g^{t}\right)\right\} \backslash\left\{b\left(g^{t}\right)-5, b\left(g^{t}\right)-3, b\left(g^{t}\right)-2, b\left(g^{t}\right)-1\right\}$.

Theorem 1.1 (Butler and Hoffman) Let $g$ and $t$ be positive integers such that $t \geq 3$, $g^{2}\left(\begin{array}{l}t \\ 2\end{array}\right) \equiv 0(\bmod 3)$, and $g(t-1) \equiv 0(\bmod 2)$. Then $\operatorname{Int}\left(g^{t}\right)=\mathcal{I}\left(g^{t}\right)$, except that

(i) $\operatorname{Int}\left(1^{9}\right)=\mathcal{I}\left(1^{9}\right) \backslash\{5,8\}$;

(ii) $\operatorname{Int}\left(2^{4}\right)=\mathcal{I}\left(2^{4}\right) \backslash\{1,4\}$;

(iii) $\operatorname{Int}\left(3^{3}\right)=\mathcal{I}\left(3^{3}\right) \backslash\{1,2,5\}$; and

(iv) $\operatorname{Int}\left(4^{3}\right)=\mathcal{I}\left(4^{3}\right) \backslash\{5,7,10\}$.

While the intersection problem has received considerable attention, the disjoint intersection problem seems not to be well-studied. The purpose of this paper is to determine completely $\operatorname{Int}_{\mathrm{d}}\left(1^{v}\right)$. This is the disjoint intersection problem for $\operatorname{STS}(v)$. Hence, we assume throughout this paper that $v \equiv 1,3(\bmod 6)$. Note that since $0,1 \in \operatorname{Int}\left(1^{v}\right)$, we also have $0,1 \in \operatorname{Int}_{\mathrm{d}}\left(1^{v}\right)$.

Define

$$
\mathcal{I}_{\mathrm{d}}\left(1^{v}\right)=\left\{\begin{array}{lll}
\{0,1, \ldots,(v-1) / 3\} & \text { if } v \equiv 1 \quad(\bmod 6), \\
\{0,1, \ldots, v / 3\} & \text { if } v \equiv 3 \quad(\bmod 6)
\end{array}\right.
$$

Since there can be at most $\lceil v / 3\rceil$ pairwise disjoint blocks in an $\operatorname{STS}(v)$, we have $\operatorname{Int}_{\mathrm{d}}\left(1^{v}\right) \subseteq$ $\mathcal{I}_{\mathrm{d}}\left(1^{v}\right)$. So in the remaining of this paper, we shall focus on showing membership of elements of $\mathcal{I}_{\mathrm{d}}\left(1^{v}\right)$ in $\operatorname{Int}_{\mathrm{d}}\left(1^{v}\right)$, rather than the other way round.

\section{Latin Squares Intersecting in a Transversal}

A Latin square of side $n$ is an $n \times n$ array with the property that every row and every column contains every element from $\{1, \ldots, n\}$ exactly once. A Latin square $A$ of side $n$ is idempotent if $A(i, i)=s_{i}$ for $1 \leq i \leq n$. A transversal in a Latin square of side $n$ is a 
set of $n$ cells, no two from the same row or from the same column, or contain the same entry. The intersection of two Latin squares $A$ and $B$ is the set of cells $(i, j)$ such that $A(i, j)=B(i, j)$. In this section, we show that for $n \neq 2,3,6$, there exists a pair of Latin squares of side $n$ intersecting in a transversal.

Two Latin squares $A$ and $B$ of side $n$ are said to be orthogonal if the $n^{2}$ ordered pairs $(A(i, j), B(i, j))$ for $1 \leq i, j \leq n$ are all distinct. The following result is well-known (see, for example, [1]).

Theorem 2.1 There exists a pair of orthogonal idempotent Latin squares of side $n$ for all $n \neq 2,3,6$.

Theorem 2.2 Let $n \neq 2,3,6$. Then there exists a pair of Latin squares of side $n$ intersecting in a transversal.

Proof. Let $A$ and $B$ be a pair of orthogonal idempotent Latin squares of side $n$. We claim that $A$ and $B$ intersect in a transversal. Obviously, the transversal formed by the main diagonal of either $A$ or $B$ is in the intersection. It remains to show that for $1 \leq i, j \leq n$, $i \neq j$, we have $A(i, j) \neq B(i, j)$. Suppose on the contrary that $A(i, j)=B(i, j)$. Then we have $(A(i, j), B(i, j))=(k, k)=(A(k, k), B(k, k))$ for some $k$. This contradicts the fact that $A$ and $B$ are orthogonal.

A $\{3\}$-GDD of type $g^{3}$ can be obtained from a Latin square $A$ of side $g$ as follows. Let $R=\left\{r_{1}, \ldots, r_{g}\right\}$ and $C=\left\{c_{1}, \ldots, c_{g}\right\}$ be the set of row and column indices of $A$, respectively. Let $S=\{1, \ldots, n\}$ be the set of entries of $A$. Define

$$
\begin{aligned}
X & =R \cup C \cup S \\
\mathcal{G} & =\{R, C, S\} \\
\mathcal{A} & =\left\{\left\{r_{i}, c_{j}, A(i, j)\right\} \mid 1 \leq i, j \leq g\right\} .
\end{aligned}
$$

Then $(X, \mathcal{G}, \mathcal{A})$ is a $\{3\}$-GDD of type $g^{3}$. From this construction, it is easy to see that Theorem 2.2 implies the following.

Corollary 2.1 For $g \neq 2,3,6$, we have $g \in \operatorname{Int}_{\mathrm{d}}\left(g^{3}\right)$.

\section{Wilson-Type Constructions}

Wilson [7] established the following fundamental construction for GDDs which has significant impact on design theory. 


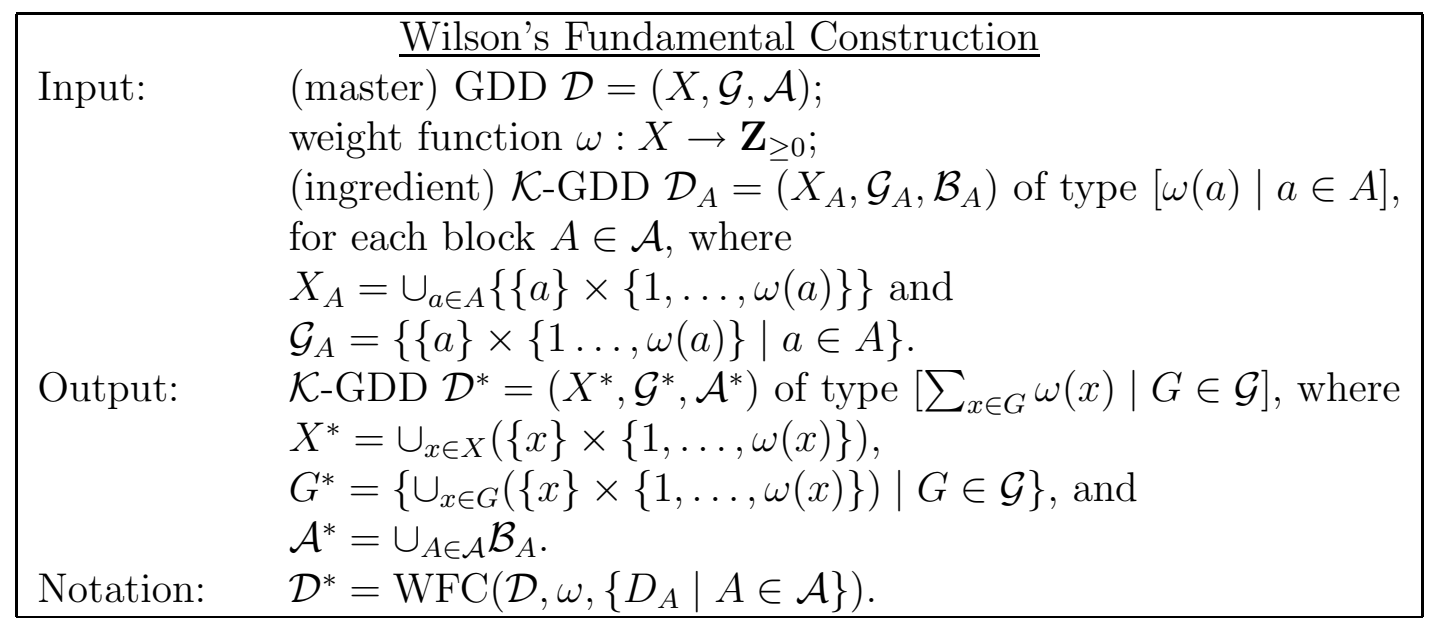

We use Wilson's fundamental construction to produce pairs of disjoint GDDs.

Theorem 3.1 Let $\mathcal{D}=(X, \mathcal{G}, \mathcal{A})$ be a $G D D$. Let $\omega: X \rightarrow \mathbf{Z}_{\geq 0}$ be a weight function. Suppose that for each block $A \in \mathcal{A}$, there exist a pair of disjoint $\{k\}$-GDDs of type $[\omega(a) \mid a \in A]$. Then there exists a pair of disjoint $\{k\}-G D D$ s of type $\left[\sum_{x \in G} \omega(x) \mid G \in \mathcal{G}\right]$.

Proof. For each block $A \in \mathcal{A}$, let $\mathcal{D}_{A}^{1}=\left(X_{A}, \mathcal{G}_{A}, \mathcal{B}_{A}^{1}\right)$ and $\mathcal{D}_{A}^{2}=\left(X_{A}, \mathcal{G}_{A}, \mathcal{B}_{A}^{2}\right)$ be a pair of disjoint $\{k\}$-GDDs of type $[\omega(a) \mid a \in A]$. Let $\mathcal{D}_{i}^{*}=\operatorname{WFC}\left(\mathcal{D}, \omega,\left\{\mathcal{D}_{A}^{i} \mid A \in \mathcal{A}\right\}\right), i=1,2$. Then $\mathcal{D}_{1}$ and $\mathcal{D}_{2}$ are $\{k\}$-GDDs of type $\left[\sum_{x \in G} \omega(x) \mid G \in \mathcal{G}\right]$.

We claim that $\mathcal{D}_{i}^{*}=\left(X^{*}, \mathcal{G}^{*}, \mathcal{A}_{i}^{*}\right), i=1,2$, is a pair of disjoint GDDs. To prove this, we have to establish the following:

$$
\begin{aligned}
& \mathcal{B}_{A}^{1} \cap \mathcal{B}_{A}^{2}=\emptyset, \text { for all } A \in \mathcal{A} \text {; and } \\
& \mathcal{B}_{A}^{1} \cap \mathcal{B}_{B}^{2}=\emptyset, \text { for all } A, B \in \mathcal{A}, A \neq B
\end{aligned}
$$

(1) follows trivially from the fact that $\left(X_{A}, \mathcal{G}_{A}, \mathcal{B}_{A}^{1}\right)$ and $\left(X_{A}, \mathcal{G}_{A}, \mathcal{B}_{A}^{2}\right)$ are disjoint, for all $A \in \mathcal{A}$. To see that (2) holds, note that for $A=\left\{a_{1}, \ldots, a_{k}\right\}$ and $B=\left\{b_{1}, \ldots, b_{k}\right\}$, blocks in $\mathcal{B}_{A}^{1}$ have the form $\left\{y_{1}, \ldots, y_{k}\right\}$, where $y_{i}=\left(a_{i}, w\right)$ for some $w \in\left\{1, \ldots, \omega\left(a_{i}\right)\right\}$, and blocks in $\mathcal{B}_{B}^{2}$ have the form $\left\{z_{1}, \ldots, z_{k}\right\}$, where $z_{i}=\left(b_{i}, w\right)$ for some $w \in\left\{1, \ldots, \omega\left(z_{i}\right)\right\}$. Hence $y_{i}=z_{j}$ is possible only if $a_{i}=b_{j}$. It follows that $\left\{y_{1}, \ldots, y_{k}\right\}=\left\{z_{1}, \ldots, z_{k}\right\}$ only if $A=B$, establishing (2).

Theorem 3.2 (Colbourn, Hoffman, and Rees) Let $g, t$, and $u$ be positive integers. There exists a $\{3\}-G D D$ of type $g^{t} u^{1}$ if and only if the following conditions are all satisfied:

(i) $t \geq 3$, or $t=2$ and $u=g$;

(ii) $u \leq g(t-1)$;

(iii) $g(t-1)+u \equiv 0(\bmod 2)$;

(iv) $g t \equiv 0(\bmod 2)$; 
(v) $g^{2}\left(\begin{array}{l}t \\ 2\end{array}\right)+g t u \equiv 0(\bmod 3)$.

Theorem 3.1 together with the existence of some $\{3\}$-GDDs provided by the above result of Colbourn, Hoffman and Rees [3] yields the following.

Corollary 3.1 For each type listed in the table below, there exist a pair of disjoint $\{3\}$ GDDs.

\begin{tabular}{|l||l|l|}
\hline \multicolumn{1}{|c|}{ Type } & $\begin{array}{c}\text { Type of Master } \\
\{3\}-G D D\end{array}$ & $\begin{array}{c}\text { Weight } \\
\text { Function }\end{array}$ \\
\hline $3^{4} 9^{1}$ & $1^{4} 3^{1}$ & $\omega(\cdot)=3$ \\
\hline $3^{6} 15^{1}$ & $1^{6} 5^{1}$ & $\omega(\cdot)=3$ \\
\hline $9^{t} 15^{1}$, & $3^{t} 5^{1}$ & $\omega(\cdot)=3$ \\
$t \equiv 0(\bmod 2), t \geq 4$ & & \\
\hline $\begin{array}{l}9^{t} 21^{1}, \\
t \equiv 0(\bmod 2), t \geq 4\end{array}$ & $3^{t} 7^{1}$ & $\omega(\cdot)=3$ \\
\hline $\begin{array}{l}9^{t} 27^{1}, \\
t \equiv 0(\bmod 2), t \geq 4\end{array}$ & $3^{t} 9^{1}$ & $\omega(\cdot)=3$ \\
\hline $\begin{array}{l}(6 s)^{t}(6 r)^{1}, \\
t \geq 3, r \leq s(t-1), s^{2}\left(\begin{array}{l}t \\
2\end{array}\right)+s t r \equiv 0(\bmod 3)\end{array}$ & $(2 s)^{t}(2 r)^{1}$ & $\omega(\cdot)=3$ \\
\hline
\end{tabular}

Proof. For each desired pair of disjoint $\{3\}$-GDDs, apply Theorem 3.1 with the listed master GDD, weight function, and use as ingredient GDDs a pair of disjoint $\{3\}$-GDDs of type $3^{3}$, which exists by Theorem 1.1. The master GDDs all exist by Theorem 3.2.

Theorem 3.3 (Filling in Groups) Suppose there exists a pair of disjoint $\mathcal{K}-G D D$ s of type $\left[g_{1}, \ldots, g_{s}\right]$. Furthermore, for each $g_{i}, 1 \leq i \leq s$, there exists a pair of $\mathcal{L}$-GDDs of type $1^{g_{i}}$ intersecting in $m_{i}$ pairwise disjoint blocks. Then there exists a pair of $(\mathcal{K} \cup \mathcal{L})-G D D s$ of type $1^{\sum_{i=1}^{s} g_{i}}$ intersecting in $\sum_{i=1}^{s} m_{i}$ pairwise disjoint blocks.

Proof. Let $(X, \mathcal{G}, \mathcal{A})$ and $(X, \mathcal{G}, \mathcal{B})$ be a pair of disjoint $\mathcal{K}$-GDDs of type $\left[g_{1}, \ldots, g_{s}\right]$, where $\mathcal{G}=\left\{G_{1}, \ldots, G_{s}\right\}$ and $\left|G_{i}\right|=g_{i}, 1 \leq i \leq s$. For each $g_{i}, 1 \leq i \leq s$, let $\left(G_{i}, \mathcal{A}_{i}\right)$ and $\left(G_{i}, \mathcal{B}_{i}\right)$ be a pair of $\mathcal{L}$-GDDs intersecting in $m_{i}$ pairwise disjoint blocks. Define

$$
\begin{aligned}
& \mathcal{D}_{1}=\left(X, \mathcal{A} \cup\left(\cup_{i=1}^{s} \mathcal{A}_{i}\right)\right) ; \text { and } \\
& \mathcal{D}_{2}=\left(X, \mathcal{B} \cup\left(\cup_{i=1}^{s} \mathcal{B}_{i}\right)\right) .
\end{aligned}
$$

$\mathcal{D}_{1}$ and $\mathcal{D}_{2}$ are clearly $(\mathcal{K} \cup \mathcal{L})$-GDDs of type $1^{\sum_{i=1}^{s} g_{i}}$. We claim that $\mathcal{D}_{1}$ and $\mathcal{D}_{2}$ intersect in $\sum_{i=1}^{s} m_{i}$ pairwise disjoint blocks. To see this, note that

(i) $\mathcal{A} \cap \mathcal{B}=\emptyset$, since $(X, \mathcal{G}, \mathcal{A})$ and $(X, \mathcal{G}, \mathcal{B})$ are disjoint GDDs;

(ii) for $1 \leq i \leq s$, we have $\mathcal{A} \cap \mathcal{B}_{i}=\emptyset$ and $\mathcal{B} \cap \mathcal{A}_{i}=\emptyset$, since any block in $\mathcal{A}$ contains at most one element from each group; and

(iii) for $1 \leq i, j \leq s, i \neq j$, we have $\mathcal{A}_{i} \cap \mathcal{B}_{j}=\emptyset$, since groups are pairwise disjoint. 
Hence, the blocks that $\mathcal{D}_{1}$ and $\mathcal{D}_{2}$ have in common are precisely the blocks in $\cup_{i=1}^{s}\left(\mathcal{A}_{i} \cap \mathcal{B}_{i}\right)$. It follows that $\mathcal{D}_{1}$ and $\mathcal{D}_{2}$ are the desired pair of GDDs intersecting in $\sum_{i=1}^{s} m_{i}$ pairwise disjoint blocks.

For $A, B \subseteq \mathbf{Z}$, we use the notation $A+B$ to denote the set $\{a+b \mid a \in A$ and $b \in B\}$. For $A_{i} \subseteq \mathbf{Z}, 1 \leq i \leq n$, we use the notation $\sum_{i=1}^{n} A_{i}$ to denote the set $\left\{a_{1}+\cdots+a_{n} \mid\right.$ $a_{i} \in A_{i}$, for $\left.1 \leq i \leq n\right\}$.

Corollary 3.2 If there exists a pair of disjoint $\{3\}-G D D$ s of type $g_{1}^{t_{1}} \cdots g_{s}^{t_{s}}$, then

$$
\operatorname{Int}_{\mathrm{d}}\left(1^{\sum_{i=1}^{s} g_{i} t_{i}}\right) \supseteq \sum_{i=1}^{s} \sum_{j=1}^{t_{i}} \operatorname{Int}_{\mathrm{d}}\left(1^{g_{i}}\right) .
$$

Proof. Apply Theorem 3.3 with GDDs intersecting in the appropriate number of pairwise disjoint blocks.

The above results are sufficient to settle the case $v \equiv 3(\bmod 6)$.

\section{Product Constructions}

We use the following Singular Direct Product construction.

\begin{tabular}{|ll|}
\hline \multirow{3}{*}{ Input: } & \multicolumn{1}{c|}{ Singular Direct Product Construction } \\
& positive integers $u, w, t ;$ \\
& for $i=1, \ldots, t,\left(\right.$ master) $\mathcal{K}$-GDDs $\mathcal{D}_{i}=\left(X_{i} \cup U, \mathcal{G}_{i}, \mathcal{A}_{i}\right)$ of type \\
& $u^{1} 1^{w_{i}}$, where $U$ is the group of size $u$ in each GDD; \\
& (ingredient) $\mathcal{K}$-GDD $\mathcal{E}=\left(\cup_{i=1}^{t} X_{i},\left\{X_{i} \mid 1 \leq i \leq t\right\}, \mathcal{B}\right)$ \\
& of type $\left[w_{i} \mid 1 \leq i \leq t\right] ;$ \\
& (fill-in) $\mathcal{K}$-GDD $\mathcal{F}=(G, \mathcal{C})$ of type $1^{u}$. \\
& $\mathcal{K}$-GDD $\mathcal{D}^{*}=\left(X^{*}, \mathcal{A}^{*}\right)$ of type $1^{u+\sum_{i=1}^{t} w_{i}}$, where \\
Output: & $X^{*}=U \cup\left(\cup_{i=1}^{t} X_{i}\right)$, and \\
& $A^{*}=\left(\cup_{i=1}^{t} \mathcal{A}_{i}\right) \cup \mathcal{B} \cup \mathcal{C}$. \\
Notation: & $\mathcal{D}^{*}=\operatorname{SDP}\left(\left\{D_{i} \mid 1 \leq i \leq t\right\}, \mathcal{E}, \mathcal{F}\right)$. \\
\hline
\end{tabular}

Lemma 4.1 Let $v \equiv 3(\bmod 6)$ and $k \equiv 0,1(\bmod 3), k \geq 3$. Then

$$
\operatorname{Int}_{\mathrm{d}}\left(1^{k(v-1)+1}\right) \supseteq \operatorname{Int}_{\mathrm{d}}\left(1^{v}\right)+\sum_{i=1}^{k-1}\left(\operatorname{Int}_{\mathrm{d}}\left(1^{v}\right) \backslash\{v / 3\}\right) .
$$

Proof. For $1 \leq i \leq k$, let $\mathcal{D}_{i}=\left(X_{i} \cup\{\infty\}, \mathcal{G}_{i}, \mathcal{A}_{i}\right)$ and $\mathcal{D}_{i}^{\prime}=\left(X_{i} \cup\{\infty\}, \mathcal{G}_{i}, \mathcal{A}_{i}^{\prime}\right)$ be a pair of $\{3\}$-GDDs of type $1^{v}$ intersecting in $m_{i}$ pairwise disjoint blocks, where $m_{1} \in \operatorname{Int}_{\mathrm{d}}\left(1^{v}\right)$ and $m_{i} \in \operatorname{Int}_{\mathrm{d}}\left(1^{v}\right) \backslash\{v / 3\}$ for $2 \leq i \leq k$. We may assume that the point $\infty$ is not contained in any blocks in the intersection of $\mathcal{D}_{i}$ and $\mathcal{D}_{i}^{\prime}, 2 \leq i \leq k$. 
Let $\mathcal{E}=\left(\cup_{i=1}^{k} X_{i},\left\{X_{i} \mid 1 \leq i \leq k\right\}, \mathcal{B}\right)$ and $\mathcal{E}^{\prime}=\left(\cup_{i=1}^{k} X_{i},\left\{X_{i} \mid 1 \leq i \leq k\right\}, \mathcal{B}^{\prime}\right)$ be a pair of disjoint $\{3\}$-GDDs of type $(v-1)^{k}$, which exists by Theorem 1.1. Let $\mathcal{F}=(\{\infty\}, \emptyset)$. Now apply the Singular Direct Product construction to obtain $\mathcal{D}_{1}^{*}=$ $\operatorname{SDP}\left(\left\{\mathcal{D}_{1}, \ldots, D_{k}\right\}, \mathcal{E}, \mathcal{F}\right)$ and $\mathcal{D}_{2}^{*}=\operatorname{SDP}\left(\left\{\mathcal{D}_{1}^{\prime}, \ldots, \mathcal{D}_{k}^{\prime}\right\}, \mathcal{E}^{\prime}, \mathcal{F}\right)$. It is easy to see that the intersection of $\mathcal{D}_{1}^{*}$ and $\mathcal{D}_{2}^{*}$ is $\cup_{i=1}^{k}\left(A_{i} \cap A_{i}^{\prime}\right)$, which contains $\sum_{i=1}^{k} m_{i}$ pairwise disjoint blocks.

Lemma 4.2 Let $s$ and $t$ be positive integers, $t \geq 3$. Then

$$
\operatorname{Int}_{\mathrm{d}}\left(1^{6 s t+1}\right) \supseteq \sum_{i=1}^{t} \operatorname{Int}_{\mathrm{d}}\left(1^{6 s+1}\right) .
$$

Proof. For $1 \leq i \leq t$, let $\mathcal{D}_{i}=\left(X_{i} \cup\{\infty\}, \mathcal{G}_{i}, \mathcal{A}_{i}\right)$ and $\mathcal{D}_{i}^{\prime}=\left(X_{i} \cup\{\infty\}, \mathcal{G}_{i}, \mathcal{A}_{i}^{\prime}\right)$ be a pair of $\{3\}$-GDDs of type $1^{6 s+1}$ intersecting in $m_{i}$ pairwise disjoint blocks, where $m_{i} \in \operatorname{Int}_{\mathrm{d}}\left(1^{6 s+1}\right)$. We may assume that the point $\infty$ is not contained in any of the intersections.

Let $\mathcal{E}=\left(\cup_{i=1}^{t} X_{i},\left\{X_{i} \mid 1 \leq i \leq t\right\}, \mathcal{B}\right)$ and $\mathcal{E}^{\prime}=\left(\cup_{i=1}^{t} X_{i},\left\{X_{i} \mid 1 \leq i \leq t\right\}, \mathcal{B}^{\prime}\right)$ be a pair of disjoint $\{3\}$-GDD of type $(6 s)^{t}$, which exists by Theorem 1.1. Let $\mathcal{F}=(\{\infty\}, \emptyset)$. Now apply the Singular Direct Product construction to obtain two $\{3\}$-GDDs of type $1^{6 s t+1}$ $\mathcal{D}_{1}^{*}=\operatorname{SDP}\left(\left\{\mathcal{D}_{i} \mid 1 \leq i \leq t\right\}, \mathcal{E}, \mathcal{F}\right)$ and $\mathcal{D}_{2}^{*}=\operatorname{SDP}\left(\left\{\mathcal{D}_{i}^{\prime} \mid 1 \leq i \leq t\right\}, \mathcal{E}^{\prime}, \mathcal{F}\right)$. It is easy to see that the intersection of $\mathcal{D}_{1}^{*}$ and $\mathcal{D}_{2}^{*}$ is $\cup_{i=1}^{t}\left(\mathcal{A}_{i} \cap \mathcal{A}_{i}^{\prime}\right)$, which contains $\sum_{i=1}^{t} m_{i}$ pairwise disjoint blocks.

Lemma 4.3 Let $r, s, t$ be positive integers such that $t \geq 3, r \leq s(t-1)$, and $s^{2}\left(\begin{array}{l}t \\ 2\end{array}\right)+$ str $\equiv 0$ $(\bmod 3)$. Then

$$
\operatorname{Int}_{\mathrm{d}}\left(1^{6 s t+6 r+1}\right) \supseteq \operatorname{Int}_{\mathrm{d}}\left(1^{6 r+1}\right)+\sum_{i=1}^{t} \operatorname{Int}_{\mathrm{d}}\left(1^{6 s+1}\right) .
$$

Proof. For $1 \leq i \leq t$, let $\mathcal{D}_{i}=\left(X_{i} \cup\{\infty\}, \mathcal{G}_{i}, \mathcal{A}_{i}\right)$ and $\mathcal{D}_{i}^{\prime}=\left(X_{i} \cup\{\infty\}, \mathcal{G}_{i}, \mathcal{A}_{i}^{\prime}\right)$ be a pair of $\{3\}$-GDDs of type $1^{6 s+1}$ intersecting in $m_{i}$ pairwise disjoint blocks, where $m_{i} \in \operatorname{Int}_{\mathrm{d}}\left(1^{6 s+1}\right)$. We may assume that the point $\infty$ is not contained in any of the intersections. Let $\mathcal{D}_{t+1}=\left(X_{t+1} \cup\{\infty\}, \mathcal{G}_{i}, \mathcal{A}_{t+1}\right)$ and $\mathcal{D}_{t+1}^{\prime}=\left(X_{t+1} \cup\{\infty\}, \mathcal{G}_{i}, \mathcal{A}_{t+1}^{\prime}\right)$ be a pair of $\{3\}$-GDDs of type $1^{6 r+1}$ intersecting in $m_{t+1}$ pairwise disjoint blocks, where $m_{t+1} \in \operatorname{Int}_{\mathrm{d}}\left(1^{6 r+1}\right)$.

Let $\mathcal{E}=\left(\cup_{i=1}^{t+1} X_{i},\left\{X_{i} \mid 1 \leq i \leq t+1\right\}, \mathcal{B}\right)$ and $\mathcal{E}^{\prime}=\left(\cup_{i=1}^{t+1} X_{i},\left\{X_{i} \mid 1 \leq i \leq t+1\right\}, \mathcal{B}^{\prime}\right)$ be a pair of disjoint $\{3\}$-GDDs of type $(6 s)^{t}(6 r)^{1}$, which exists by Corollary 3.1. Let $\mathcal{F}=(\{\infty\}, \emptyset)$. Now apply the Singular Direct Product construction to obtain two $\{3\}$ GDDs of type $1^{6 s t+6 r+1} \mathcal{D}_{1}^{*}=\operatorname{SDP}\left(\left\{\mathcal{D}_{i} \mid 1 \leq i \leq t+1\right\}, \mathcal{E}, \mathcal{F}\right)$ and $\mathcal{D}_{2}^{*}=\operatorname{SDP}\left(\left\{\mathcal{D}_{i}^{\prime} \mid 1 \leq\right.\right.$ $\left.i \leq t+1\}, \mathcal{E}^{\prime}, \mathcal{F}\right)$. It is easy to see that the intersection of $\mathcal{D}_{1}^{*}$ and $\mathcal{D}_{2}^{*}$ is $\cup_{i=1}^{t+1}\left(\mathcal{A}_{i} \cap \mathcal{A}_{i}^{\prime}\right)$, which contains $\sum_{i=1}^{t+1} m_{i}$ pairwise disjoint blocks. 


\section{Direct Constructions}

In this section, we determine $\operatorname{Int}_{\mathrm{d}}\left(1^{v}\right)$ for some small values of $v$.

Lemma 5.1 The following equalities hold:

(i) $\operatorname{Int}_{\mathrm{d}}\left(1^{1}\right)=\{0\}$;

(ii) $\operatorname{Int}_{\mathrm{d}}\left(1^{3}\right)=\{1\}$;

(iii) $\operatorname{Int}_{\mathrm{d}}\left(1^{7}\right)=\{0,1\}$;

(iv) $\operatorname{Int}_{\mathrm{d}}\left(1^{9}\right)=\{0,1,3\}$; and

(v) for $v \equiv 1,3(\bmod 6), 13 \leq v \leq 31, \operatorname{Int}_{\mathrm{d}}\left(1^{v}\right)=\mathcal{I}_{\mathrm{d}}\left(1^{v}\right)$.

\subsection{Proof of Lemma 5.1}

(i) and (ii) hold trivially and recall that $0,1 \in \operatorname{Int}_{\mathrm{d}}\left(1^{v}\right)$ for $v \equiv 1,3(\bmod 6), v \geq 7$. For $7 \leq v \leq 31$, we have the following table.

\begin{tabular}{|r|l|l|}
\hline$v$ & $\begin{array}{l}\text { Elements } \\
\text { in } \operatorname{Int}_{\mathrm{d}}\left(1^{v}\right)\end{array}$ & Authority \\
\hline 7 & 0,1 & Trivial \\
\hline 9 & $0,1,3$ & Corollary 3.2: $\operatorname{Int}_{\mathrm{d}}\left(1^{9}\right) \supseteq \sum_{i=1}^{3} \operatorname{Int}_{\mathrm{d}}\left(1^{3}\right)$ \\
\hline 13 & 0,1 & Trivial \\
\hline 15 & $0,1,5$ & Corollary 3.2: $\operatorname{Int}_{\mathrm{d}}\left(1^{15}\right) \supseteq \sum_{i=1}^{5} \operatorname{Int}_{\mathrm{d}}\left(1^{3}\right)$ \\
\hline 19 & $0,1,2,3$ & Lemma 4.2: $\operatorname{Int}_{\mathrm{d}}\left(1^{19}\right) \supseteq \sum_{i=1}^{3} \operatorname{Int}_{\mathrm{d}}\left(1^{7}\right)$ \\
\hline 21 & $0,1,2,3,4,5,7$ & $\begin{array}{l}\text { Corollary 3.2: } \operatorname{Int}_{\mathrm{d}}\left(1^{21}\right) \supseteq \sum_{i=1}^{3} \operatorname{Int}_{\mathrm{d}}\left(1^{7}\right) ; \\
\text { Corollary 3.2: } \operatorname{Int}_{\mathrm{d}}\left(1^{21}\right) \supseteq \operatorname{Int}_{\mathrm{d}}\left(1^{9}\right)+\sum_{i=1}^{4} \operatorname{Int}_{\mathrm{d}}\left(1^{3}\right)\end{array}$ \\
\hline 25 & $0,1,2,3,4,5$ & Lemma 4.1: $\operatorname{Int}_{\mathrm{d}}\left(1^{25}\right) \supseteq \operatorname{Int}_{\mathrm{d}}\left(1^{9}\right)+\sum_{i=1}^{2}\left(\operatorname{Int}_{\mathrm{d}}\left(1^{9}\right) \backslash\{3\}\right)$ \\
\hline 27 & $0,1,2,3,4,5,6,7,9$ & Corollary 3.2: $\operatorname{Int}_{\mathrm{d}}\left(1^{27}\right) \supseteq \sum_{i=1}^{3} \operatorname{Int}_{\mathrm{d}}\left(1^{9}\right)$ \\
\hline 31 & $0,1,2,3,4,5,6,7$ & Lemma 4.3: $\operatorname{Int}_{\mathrm{d}}\left(1^{31}\right) \supseteq \operatorname{Int}_{\mathrm{d}}\left(1^{13}\right)+\sum_{i=1}^{3} \operatorname{Int}_{\mathrm{d}}\left(1^{7}\right)$ \\
\hline
\end{tabular}

Let $\mathcal{S}_{v}, v \in\{13,15,19,21,25,27,31\}$ be the $\operatorname{STS}(v)$ 's given in Appendix A. For an $\operatorname{STS}(v) \mathcal{S}_{v}=(X, \mathcal{B})$ and a permutation $\pi: X \rightarrow X, \pi$ acts on $\mathcal{S}_{v}$ by canonical extension as follows: $\pi\left(\mathcal{S}_{v}\right)=\{\{\pi(a), \pi(b), \pi(c)\} \mid\{a, b, c\} \in \mathcal{B}\}$. The permutations $\pi$ listed below is such that $\mathcal{S}_{v}$ and $\pi\left(\mathcal{S}_{v}\right)$ intersect in $m$ pairwise disjoint blocks, where $m$ is a number whose membership in $\operatorname{Int}_{\mathrm{d}}\left(1^{v}\right)$ has not been established in the table above. 


\begin{tabular}{|c|c|c|}
\hline$v$ & $\begin{array}{l}\text { Elements } \\
\text { in } \operatorname{Int}_{\mathrm{d}}\left(1^{v}\right)\end{array}$ & $\pi$ \\
\hline 13 & $\begin{array}{l}2 \\
3 \\
4\end{array}$ & 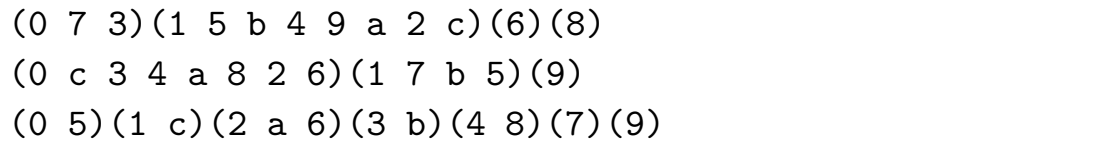 \\
\hline 15 & $\begin{array}{l}2 \\
3 \\
4\end{array}$ & 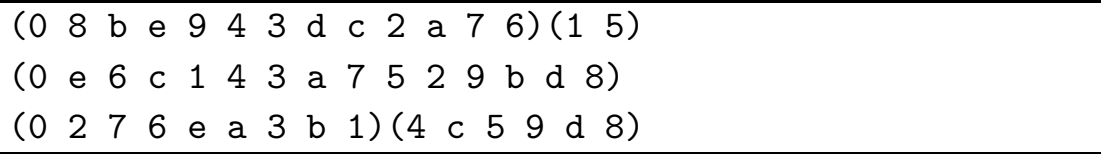 \\
\hline 19 & $\begin{array}{l}4 \\
5\end{array}$ & 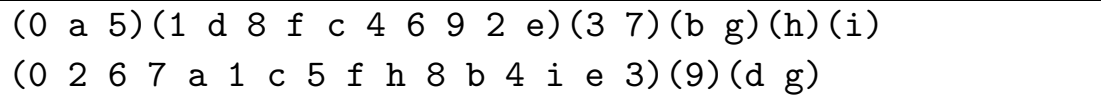 \\
\hline 25 & 6 & 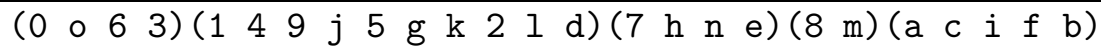 \\
\hline
\end{tabular}

Any two blocks of an $\operatorname{STS}(7)$ intersect in a point. So $2 \notin \operatorname{Int}_{\mathrm{d}}\left(1^{7}\right)$. In an $\operatorname{STS}(9)$ $(X, \mathcal{B})$, if $A, B \in \mathcal{B}$ and $A \cap B=\emptyset$, then $X \backslash(A \cup B)$ is a block in $\mathcal{B}$. So $2 \notin \operatorname{Int}_{\mathrm{d}}\left(1^{9}\right)$. For each of the remaining possible disjoint intersection size $m \in \operatorname{Int}_{\mathrm{d}}\left(1^{v}\right)$, we exhibit an $\operatorname{STS}(v) \mathcal{D}_{v}$, listed in Appendix B, whose intersection with $\mathcal{S}_{v}$ contains $m$ pairwise disjoint blocks.

\begin{tabular}{|c|l|l|}
\hline$v$ & $\begin{array}{l}\text { Remaining values } \\
\text { in } \operatorname{Int}_{\mathrm{d}}\left(1^{v}\right)\end{array}$ & $\mathcal{T}_{v}$ \\
\hline 19 & 6 & $\mathcal{T}_{1}$ \\
\hline 21 & 6 & $\mathcal{T}_{2}$ \\
\hline 25 & 7 & $\mathcal{T}_{3}$ \\
& 8 & $\mathcal{T}_{4}$ \\
\hline 27 & 8 & $\mathcal{T}_{5}$ \\
\hline 31 & 8 & $\mathcal{T}_{6}$ \\
& 9 & $\mathcal{T}_{7}$ \\
& 10 & $\mathcal{T}_{8}$ \\
\hline
\end{tabular}

\section{Piecing Things Together}

We first deal with the easier case of $v \equiv 3(\bmod 6)$.

\subsection{The Case $v \equiv 3 \bmod 6$}

\subsection{1 $v \equiv 3(\bmod 18)$}

By Theorem 1.1, there exists a pair of disjoint $\{3\}$-GDDs of type $13^{3}$. Corollary 3.2 then gives $\operatorname{Int}_{\mathrm{d}}\left(1^{39}\right) \supseteq \sum_{i=1}^{3} \operatorname{Int}_{\mathrm{d}}\left(1^{13}\right)=\sum_{i=1}^{3}\{0, \ldots, 4\}=\{0, \ldots, 12\}$. By Theorem 1.1, there also exists a pair of disjoint $\{3\}$-GDDs of type $3^{13}$. Corollary 3.2 now gives $\operatorname{Int}_{\mathrm{d}}\left(1^{39}\right) \supset \sum_{i=1}^{13} \operatorname{Int}_{\mathrm{d}}\left(1^{3}\right)=\sum_{i=1}^{13}\{1\}=\{13\}$. Hence $\operatorname{Int}_{\mathrm{d}}\left(1^{39}\right)=\mathcal{I}_{\mathrm{d}}\left(1^{39}\right)$. 
For $v \geq 57$, consider a pair of disjoint $\{3\}$-GDDs of type $9^{t} 21^{1}$, where $t=(v-21) / 9$, existence of which is provided by Corollary 3.1. Now apply Corollary 3.2 to obtain

$$
\begin{aligned}
\operatorname{Int}_{\mathrm{d}}\left(1^{v}\right) & \supseteq \operatorname{Int}_{\mathrm{d}}\left(1^{21}\right)+\sum_{i=1}^{t} \operatorname{Int}_{\mathrm{d}}\left(1^{9}\right) \\
& =\{0, \ldots, 7\}+\sum_{i=1}^{t}\{0,1,3\} \\
& =\{0, \ldots, 3 t+7\} \\
& =\mathcal{I}_{\mathrm{d}}\left(1^{v}\right) .
\end{aligned}
$$

This establishes the following.

Lemma 6.1 $\operatorname{Int}_{\mathrm{d}}\left(1^{v}\right)=\mathcal{I}_{\mathrm{d}}\left(1^{v}\right)$ for $v \equiv 3(\bmod 18), v \geq 39$.

\subsection{2 $v \equiv 9(\bmod 18)$}

By Theorem 1.1, there exists a pair of disjoint $\{3\}$-GDDs of type $15^{3}$. Corollary 3.2 then gives $\operatorname{Int}_{\mathrm{d}}\left(1^{45}\right) \supseteq \sum_{i=1}^{3} \operatorname{Int}_{\mathrm{d}}\left(1^{15}\right)=\sum_{i=1}^{3}\{0, \ldots, 5\}=\{0, \ldots, 15\}=\mathcal{I}_{\mathrm{d}}\left(1^{45}\right)$.

For $v \geq 63$, consider a pair of disjoint $\{3\}$-GDDs of type $9^{t} 27^{1}$, where $t=(v-27) / 9$, existence of which is provided by Corollary 3.1. Now apply Corollary 3.2 to obtain

$$
\begin{aligned}
\operatorname{Int}_{\mathrm{d}}\left(1^{v}\right) & \supseteq \operatorname{Int}_{\mathrm{d}}\left(1^{27}\right)+\sum_{i=1}^{t} \operatorname{Int}_{\mathrm{d}}\left(1^{9}\right) \\
& =\{0, \ldots, 9\}+\sum_{i=1}^{t}\{0,1,3\} \\
& =\{0, \ldots, 3 t+9\} \\
& =\mathcal{I}_{\mathrm{d}}\left(1^{v}\right) .
\end{aligned}
$$

This establishes the following.

Lemma 6.2 $\operatorname{Int}_{\mathrm{d}}\left(1^{v}\right)=\mathcal{I}_{\mathrm{d}}\left(1^{v}\right)$ for $v \equiv 9(\bmod 18), v \geq 45$.

\subsection{3 $v \equiv 15(\bmod 18)$}

By Corollary 3.1, there exists a pair of disjoint $\{3\}$-GDDs of type $3^{6} 15^{1}$. Corollary 3.2 then gives $\operatorname{Int}_{\mathrm{d}}\left(1^{33}\right) \supseteq \operatorname{Int}_{\mathrm{d}}\left(1^{15}\right)+\sum_{i=1}^{6} \operatorname{Int}_{\mathrm{d}}\left(1^{3}\right)=\{0, \ldots, 5\}+\{6\}=\{6, \ldots, 11\}$. Also, by Lemma 4.1, we have $\operatorname{Int}_{\mathrm{d}}\left(1^{33}\right) \supseteq \operatorname{Int}_{\mathrm{d}}\left(1^{9}\right)+\sum_{i=1}^{3}\left(\operatorname{Int}_{\mathrm{d}}\left(1^{9}\right) \backslash\{3\}\right)=\{0,1,3\}+\sum_{i=1}^{3}\{0,1\}=$ $\{0,1,2,3,4,5,6\}$. Hence we have $\operatorname{Int}_{\mathrm{d}}\left(1^{33}\right)=\mathcal{I}_{\mathrm{d}}\left(1^{33}\right)$. 
For $v \geq 51$, consider a pair of disjoint $\{3\}$-GDDs of type $9^{t} 15^{1}$, where $t=(v-15) / 9$, whose existence is provided by Corollary 3.1. Now apply Corollary 3.2 to obtain

$$
\begin{aligned}
\operatorname{Int}_{\mathrm{d}}\left(1^{v}\right) & \supseteq \operatorname{Int}_{\mathrm{d}}\left(1^{15}\right)+\sum_{i=1}^{t} \operatorname{Int}_{\mathrm{d}}\left(1^{9}\right) \\
& =\{0, \ldots, 5\}+\sum_{i=1}^{t}\{0,1,3\} \\
& =\{0, \ldots, 3 t+5\} \\
& =\mathcal{I}_{\mathrm{d}}\left(1^{v}\right) .
\end{aligned}
$$

This establishes the following.

Lemma 6.3 $\operatorname{Int}_{\mathrm{d}}\left(1^{v}\right)=\mathcal{I}_{\mathrm{d}}\left(1^{v}\right)$ for $v \equiv 15(\bmod 18), v \geq 33$.

\subsection{The Case $v \equiv 1(\bmod 6)$}

\subsection{1 $v \equiv 1(\bmod 18)$}

Let $s=2, t=3$. Then Lemma 4.2 gives $\operatorname{Int}_{\mathrm{d}}\left(1^{37}\right) \supseteq \sum_{i=1}^{3} \operatorname{Int}_{\mathrm{d}}\left(1^{13}\right)=\sum_{i=1}^{3}\{0, \ldots, 4\}=$ $\{0, \ldots, 12\}=\mathcal{I}_{\mathrm{d}}\left(1^{37}\right)$.

For $v \geq 55$, let $s=3, t=(v-1) / 18$. Now apply Lemma 4.2 to obtain

$$
\begin{aligned}
\operatorname{Int}_{\mathrm{d}}\left(1^{v}\right) & \supseteq \sum_{i=1}^{t} \operatorname{Int}_{\mathrm{d}}\left(1^{19}\right) \\
& =\sum_{i=1}^{t}\{0, \ldots, 6\} \\
& =\{0, \ldots, 6 t\} \\
& =\mathcal{I}_{\mathrm{d}}\left(1^{v}\right) .
\end{aligned}
$$

This establishes the following.

Lemma 6.4 $\operatorname{Int}_{\mathrm{d}}\left(1^{v}\right)=\mathcal{I}_{\mathrm{d}}\left(1^{v}\right)$ for $v \equiv 1(\bmod 18), v \geq 37$.

\subsection{2 $v \equiv 7(\bmod 18)$}

Let $v \geq 43$. Let $w=(v+2) / 3$. Then $w \equiv 3(\bmod 6)$ and $w \geq 15$. Now apply Corollary 4.1 to obtain

$$
\begin{aligned}
\operatorname{Int}_{\mathrm{d}}\left(1^{v}\right) & \left.\left.\supseteq \operatorname{Int}_{\mathrm{d}}\left(1^{w}\right)+\left(\operatorname{Int}_{\mathrm{d}}\left(1^{w}\right) \backslash\{w / 3\}\right)+\left(\operatorname{Int}_{\mathrm{d}}\left(1^{w}\right) \backslash\right\} w / 3\right\}\right) \\
& =\{0, \ldots, w / 3\}+\{0, \ldots, w / 3-1\}+\{0, \ldots, w / 3-1\} \\
& =\{0, \ldots, w-2\} \\
& =\{0, \ldots,(v-1) / 3-1\} .
\end{aligned}
$$


To show that $(v-1) / 3 \in \operatorname{Int}_{\mathrm{d}}\left(1^{v}\right)$, mimic the proof of Lemma 4.1 but with $m_{1}=m_{2}=$ $m_{3}=0$ and replace the ingredient $\{3\}$-GDD of type $(w-1)^{3}$ with a pair of $\{3\}$-GDDs of type $(w-1)^{3}$ intersecting in $w-1$ disjoint blocks, existence of which is provided by Corollary 2.1. This establishes the following.

Lemma 6.5 $\operatorname{Int}_{\mathrm{d}}\left(1^{v}\right)=\mathcal{I}_{\mathrm{d}}\left(1^{v}\right)$ for $v \equiv 7(\bmod 18), v \geq 43$.

\subsection{3 $v \equiv 13(\bmod 18)$}

Let $s=2$ and $t=4$. Then Lemma 4.2 gives $\operatorname{Int}_{\mathrm{d}}\left(1^{49}\right) \supseteq \sum_{i=1}^{4} \operatorname{Int}_{\mathrm{d}}\left(1^{13}\right)=\sum_{i=1}^{4}\{0, \ldots, 4\}=$ $\{0, \ldots, 16\}=\mathcal{I}_{\mathrm{d}}\left(1^{49}\right)$.

For $v \geq 67$, let $t=(v-13) / 18$. Then $t \geq 3$. Now apply Corollary 4.3 with $r=2$ and $s=3$ to obtain

$$
\begin{aligned}
\operatorname{Int}_{\mathrm{d}}\left(1^{v}\right) & =\operatorname{Int}_{\mathrm{d}}\left(1^{18 t+13}\right) \\
& \supseteq \operatorname{Int}_{\mathrm{d}}\left(1^{13}\right)+\sum_{i=1}^{t} \operatorname{Int}_{\mathrm{d}}\left(1^{19}\right) \\
& =\{0, \ldots, 4\}+\sum_{i=1}^{t}\{0, \ldots, 6\} \\
& =\{0, \ldots, 6 t+4\} \\
& =\mathcal{I}_{\mathrm{d}}\left(1^{v}\right) .
\end{aligned}
$$

This establishes the following.

Lemma 6.6 $\operatorname{Int}_{\mathrm{d}}\left(1^{v}\right)=\mathcal{I}_{\mathrm{d}}\left(1^{v}\right)$ for $v \equiv 13(\bmod 18), v \geq 49$.

\section{Conclusion}

Lemmas 6.1 to 6.6 shows that $\operatorname{Int}_{\mathrm{d}}\left(1^{v}\right)=\mathcal{I}_{\mathrm{d}}\left(1^{v}\right)$ for all $v \equiv 1,3(\bmod 6), v \geq 33$. Lemma

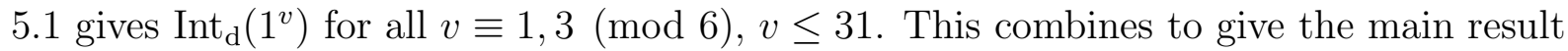
of this paper below.

Theorem 7.1 Let $v \equiv 1,3(\bmod 6)$. Then $\operatorname{Int}_{\mathrm{d}}\left(1^{v}\right)=\mathcal{I}_{\mathrm{d}}\left(1^{v}\right)$, except that

(i) $\operatorname{Int}_{\mathrm{d}}\left(1^{7}\right)=\{0,1\}$; and

(ii) $\operatorname{Int}_{\mathrm{d}}\left(1^{9}\right)=\{0,1,3\}$.

If there exists a pair of $\operatorname{STS}(v)$ intersecting in $t$ pairwise disjoint blocks, we can consider these $t$ pairwise disjoint blocks as groups of a pair of disjoint $\{3\}$-GDDs of type $3^{t} 1^{v-t}$, and vice versa. Hence, Theorem 7.1 is equivalent to the following.

Theorem 7.2 Let $3 t+r \equiv 1,3(\bmod 6), 3 t+r \geq 13$. Then there exists a pair of disjoint $\{3\}-G D D$ s of type $3^{t} 1^{r}$. 


\section{References}

[1] Abel, R. J. R., Brouwer, A. E., Colbourn, C. J., And Dinitz, J. H. Mutually orthogonal Latin squares (MOLS). In The CRC Handbook of Combinatorial Designs, C. J. Colbourn and J. H. Dinitz, Eds. CRC Press, Boca Raton, 1996, ch. II.2, pp. 111142 .

[2] Butler, R. A. R., And Hoffman, D. G. Intersections of group divisible triple systems. Ars Combin. 34 (1992), 268-288.

[3] Colbourn, C. J., Hoffman, D. G., and Rees, R. A new class of group divisible designs with block size three. J. Combin. Theory Ser. A 59 (1992), 73-89.

[4] Fu, H. L. On the construcion of certain types of Latin squares having prescribed intersections. PhD thesis, Department of Discrete and Statistical Sciences, Auburn University, Auburn, Alabama, 1980.

[5] Hoffman, D. G., And Lindner, C. C. The flower intersection problem for Steiner triple systems. Ann. Discrete Math. 34 (1987), 243-258.

[6] Lindner, C. C., And Rosa, A. Steiner triple systems with a prescribed number of triples in common. Canad. J. Math. 27 (1975), 1166-1175.

[7] Wilson, R. M. An existence theory for pairwise balanced designs I: Composition theorems and morphisms. J. Combin. Theory Ser. A 13 (1971), 220-245. 


\title{
A Some Small Steiner Triple Systems
}

For space efficiency, we list a block $\{a, b, c\}$ vertically as $\underset{c}{b}$.

\author{
A.1 An STS(13) \\ 00000011111222223334445556 \\ 13579b3469a3467867868a7897 \\ 2468ac578bc95acbbacc9bbac9
}

\section{A.2 An STS(15)}

00000001111112222223333444455556666

13579bd3478bc34789c789a789a789a78ab

2468ace569ade65abedcdbeecdbbecdd9ce

\section{A.3 An STS(19)}

$00000000011111111222222223333334444455555666777888999 a b c f$ 13456abce345678ac345678ad4689bg67acd678bd79f abe9beacdhedg 2789dgfihh9ibgdeffbeh9icg5acedieifghchagf8gidcffhgbhiiifh

\section{A.4 An STS(21)}

$0000000000111111111222222222333333344444455555566667778888999 a a b b c c d e f$ 13456789af345679bdh345678bcf4679abc678adh678bde79dgabe9acgaf ieicedhgjg 2ejcbhidgk8egafcikjd9ajkehgi5fjghkicgbkfii9kfjh8ehkcdihdfjbjkfjjgekikh

\section{A.5 An STS(25)}

0000000000001111111111122222222222333333333444444445555555566666677777 1345689degin34567bcdefh345679abdf j4689abgik679ceghk679bcegj79abchabcef 27baf jchlkmoma8o9jniglkc8hkoeglmin5elhfdjnodlfimojnldknf imo8migjnkigjn

788888889999aaaabbbccdddeffhil h9abcefgadgicejmcekdkfglhgjijm mndhmkoibjlohnloofmelknoohmlkn

\section{A.6 An STS(27)}

0000000000000111111111111222222222222333333333344444444455555555566666 13456789abdeh3456789abdfg3456789acef j4678acdfkn6789abijp6789abcde79adl 2pgnkfqcmiljolhpecnjiqmokhdgimbpkqonl5be9ogijmqcnfkemloqjlihqomkf8gfhq 
6677777788888899999aaabbbbbccccdeeefffggghhiikl mn9abdghacdehkadeincdhcdef jdfiljghkgimjlmjkjoom opqjkpoigjomplbflmopnlhgnlpeknoqiqphqpnpqmnkpqn

\section{A.7 An STS(31)}

0000000000000001111111111111122222222222222333333333333444444444445555 13456789acfhijn34567abcdghjms3456789bdefiln4679abcdef jn678bcdf ghmn6789 2plbergdotkmqus89elfkoqnirptuoahjkcugqptmsr5mtgiurhklsqrqjeotiskpuiosm

$5555555666666666777777778888888889999999 a a a a a a b b b b b b c c c c d d d d e e e e e f f g g$ acdfglp79abcfghq9abcdijp9abdehikmachijkncefhnpcdfhjkdfgmfgikfghiorgjjn rnjukqt8odpknusteglhmsnufuqrlntpobsqlrtpjqmltsisrtmnepluopulsmjntuhqto

ghhiijkkklllmor qiojolmoqmptqps rpukrorsunrusqt 


\section{B A Few More Small Steiner Triple Systems}

\section{B.1 An STS(19)}

B.1.1 $\mathcal{T}_{1}$ $000000000111111112222222233333344444555556666677788899 \mathrm{bcf}$ 1345678ag34578abd3459abde479bcf78acd68abd79ace9ce9abaehdg 2d9ebfhci6e9higcf867cfghi5ahegibfihggchfi8idfhdiggedbfieh

\section{B.2 An STS(21)}

\section{B.2.1 $\mathcal{T}_{2}$}

$0000000000111111111222222222333333344444455555566666777788899 a a b b c c d e f$ 1345689abd345678cfg345789abc4789abd6789bi689cde7abcg9adh9efaeefhjdgihg 2ig7jkhcefeha9bdikj6kiejfdgh5fhkgcjecadfjkbgjhf8hdfijigkcgibikjikekkjh

\section{B.3 Two STS(25)'s}

\section{B.3.1 $\mathcal{T}_{3}$}

0000000000001111111111122222222222333333333444444445555555566666677777 13456789abcd345689adef j345689abefn4689abekl68abcdh16789abeg7acdhm9abdf 2johemglkinf i7nbhgclkmo7icjdkmghlo5f cdhmgno9f jegknmijmfldok8olgknegcok

77888889999aabbbccccddeeffgggi

hl9abekachjdefhkdfhkhiijgiijmj inoijnlbimnnfnloejomjmlmhonlok

\section{B.3.2 $\mathcal{T}_{4}$}

0000000000001111111111122222222222333333333444444445555555566666667777 13456789abf i34569cdegjk345789abcef467abdefh6789aehn89abcdeh7bdf ghm9abd 2ndgckljehmo8b7afhniomlgi6mhdlonjk59cimokljkfjcglmonijfokml8ejnliolojh

778888889999aaabbbccccdefghij1 eg9abdefaegkdhkcdidfgiifgjkjlm niockmgibhmnfnmlgnejkmlohnokon

\section{B.4 An STS(27)}

\section{B.4.1 $\mathcal{T}_{5}$}

0000000000000111111111111222222222222333333333344444444455555555566666 1345689abcdgk3456789abimn345679abdfgo46789abceh679bdeghm689abcdgk79bdg 2ml7f jehnqoipkcfjhlgedqopn8hcekjlimqp5lpqodgfijainkjfopqeoipjnmlq8hoqn 
66777777788888889999aaaabbbccccddeeeeefffghiijl ik9abcdfj9abdghmadflcfilcfhdhijhkghjkpgkljojnnm pmmkqognlcnefkipbpjqgqmopimeklmnlmlonqhoppqkoqn

\section{B.5 Three STS(31)'s}

\section{B.5.1 $\mathcal{T}_{6}$}

0000000000000001111111111111122222222222222333333333333444444444445555 1345789abceghjp3456789acdegio345678bcdeghim478abdegjlqs6789abcei jp6789

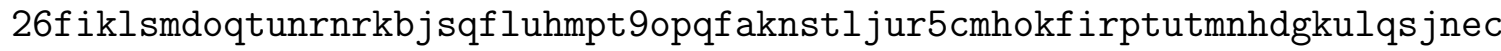
$5555555666666666677777777888888889999999 a a a a a a b b b b b b c c c c c d d d d e e e f f f f f$ abdfhmt79acdefkmn9abdghlr9bcdhikpadef gkmcijknpcehinqdfgimfhijiklogknpr glrsqou8lohpgiusriesqoputtf jgroqubonjrpusqlrutujmtpretprqmlntmsrphloqu ggghhhijjklllno jkqiknjmommosqr snusotkputnqtss

\section{B.5.2 $\mathcal{T}_{7}$}

0000000000000001111111111111122222222222222333333333333444444444445555 13456789abdehil345689abcdfgjn345679abdehmqs4678abfghlno69abceghikr6789 2qfunjmsctgokprs7qoklmeihuprtk8nclfgptjioru59redcimjuptldtjpnumqostajo $55555556666666667777777778888888889999999 a a a a a a b b b b b c c c c c c d d d d e e e e f f f$ bcdefhk7abdeghkm9bcdefhin9abcdfgiqacegijmefhikldhknodfhjmtijmnghkrgkno gsrimpl8qipf jursumgkpqotshrlnupsotbkqtrnpujnspofrsquelqorulsqolsmthtrs ggghiijjjkllopp ikoljmlmqnmqprs nqrtkuptuunsqut

\section{B.5.3 $\mathcal{T}_{8}$}

0000000000000001111111111111122222222222222333333333333444444444445555 1345678abcdeijk3456789bcef gmn34567acdfijlnq4678abcdgioq679bcdef jpr6789 29hqgmt sunofplrkahipdoqlut jsre8gb9mhkpturos5plfthj smnrumnitglkoqsurslp $55555556666666667777777788888888899999999 a a a a a a b b b b b b c c c c d d d d e e e f f f g g$ abdefik79acdefhnabcfghjk9abceghjkacdefhjlcdghkpefgjlmdfpshinpghjmginkl octnjum8slojtqkuedquritomqnripsoubkgqruntifujnrpkirsoemtumrquolsrhlssq ghhhiiijkkllmor nnoqjmomlqmotps tptrkqspptnuuqt 